\title{
Niñez migrante en Colombia: grises del aclamado estatuto temporal de protección
}

\section{Migrant Children in Colombia: Greys of the Applauded Temporary Protection Statute}

\section{Gracy Pelacani}

Docente de la Universidad de los Andes

Bogotá, Colombia

g.pelacani@uniandes.edu.co

ORCID: 0000-0002-8801-8752

Artículo de investigación

DOI: https://doi.org/10.32719/26312484.2022.37.2

Fecha de recepción: 29 de junio de 2021

Fecha de revisión: 27 de agosto de 2021

Fecha de aceptación: 15 de septiembre de 2021

Fecha de publicación: 3 de enero de 2022 


\section{RESUMEN}

En el presente trabajo se analiza críticamente el enfoque de niñez del Estatuto Temporal de Protección para Migrantes Venezolanos adoptado por Colombia en marzo de 2021, para preguntarse si este responde a las necesidades de protección de los niños venezolanos. El Estatuto reconoce que contar con un estatus migratorio regular es un paso previo y necesario para la garantía plena de sus derechos. Por lo tanto, en un primer momento, se considera cómo el Estado colombiano respondió a algunos entre los principales retos de protección de la niñez venezolana en Colombia y se evidencian los vacíos que permanecen en estas actuaciones. Posteriormente, se profundiza en la forma en que el Estatuto pretende garantizar el acceso a un estatus migratorio regular a los niños y, con ello, la protección y garantía de sus derechos. El trabajo concluye que el Estatuto es un avance necesario pero insuficiente para la protección de los derechos de los niños migrantes en Colombia. Esto es así porque sigue perpetuando una visión limitada y sesgada de la migración y promueve la creación de un doble estándar de protección que perjudica a niños de nacionalidades diferentes a la venezolana.

Palabras Clave: Estatuto Temporal de Protección para Migrantes Venezolanos; niñez migrante; política migratoria; regularización migratoria; acceso a derechos; doble estándar; Colombia; Venezuela.

\section{ABSTRACT}

This article critically analyses the child rights-based approach of the Temporary Protection Statute for Venezuelan Migrants adopted by Colombia in March 2021, to question if it responds to the protection needs of Venezuelan children. The Statute recognizes that having a regular migratory status is a prior and necessary step for the full guarantee of children rights. Therefore, at first, the study considers how the Colombian State has responded to some of the main protection challenges of Venezuelan children in Colombia and the gaps that remains in the actions undertaken. Then, it delves into the way in which the Temporary Protection Statute aims at granting access to a regular migratory status and the protection of the rights of Venezuelan children in Colombia. This article concludes that the Temporary Protection Status is a necessary but insufficient advance for the protection of migrant children rights. This is so because it perpetuates a limited and biased view of migration and promotes the creation of a double standard of protection that prejudices children of nationalities other than the Venezuelan.

KeYwords: Temporary Protection Statute for Venezuelan Migrants; migrant children; migration policy; migratory regularization; access to rights; double standard; Colombia; Venezuela. 


\section{INTRODUCCIÓN}

"E n el contexto de la migración internacional, los niños pueden encontrarse en una situación de doble vulnerabilidad como niños y como niños afectados por la migración...". ${ }^{1}$ Esta será una condición de triple vulnerabilidad, toda vez que ellos, sus padres o representantes legales se encuentran en situación migratoria irregular. ${ }^{2}$ Así las cosas, la inclusión de un enfoque de niñez en las políticas públicas en materia migratoria es una tarea inaplazable para los Estados, en la cual se deberá anteponer a cualquier otra consideración que los niños en situación de movilidad humana son, ante todo, niños. En este sentido, la garantía de sus derechos prevalece y se antepone a los objetivos de control y gestión de la migración. ${ }^{3}$

En este contexto, el 8 de febrero de 2021, el presidente de la República de Colombia anunció la adopción del Estatuto Temporal de Protección para Migrantes Venezolanos (en adelante ETPV o Estatuto). ${ }^{4}$ El Alto Comisionado de Naciones Unidas para los Refugiados, Filippo Grandi, que acompañó el anuncio, definió el Estatuto como "el gesto humanitario más importante que se ha hecho en este continente desde 1984, cuando se firmó la Declaración de Cartagena". ${ }^{5}$ El ETPV busca responder a dos limitantes que, hasta el momento, presentaba la política pública en materia migratoria del Estado colombiano: por un lado, la falta de datos confiables sobre la población migrante de nacionalidad venezolana presente en Colombia, lo cual impacta profundamente en la formulación de la política pública en materia migratoria. Por el otro, el hecho de que más de la mitad de esta población se encuentra en situación migratoria irregular, siendo este el primer obstáculo para la garantía de sus derechos y acceso a servicios básicos.

1. ONU, Comité de los Derechos del Niño y Comité de Protección de los Derechos de Todos los Trabajadores Migratorios y de sus Familiares, Observación general conjunta núm. 3 (2017) del Comité de Protección de los Derechos de Todos los Trabajadores Migratorios y de sus Familiares y núm. 22 (2017) del Comité de los Derechos del Niño sobre los principios generales relativos a los derechos humanos de los niños en el contexto de la migración internacional, 16 de noviembre de 2017, CMW/C/GC/3-CRC/C/ GC/22, párr. 3 .

2. ONU, UNICEF, La regularización migratoria como condición esencial para la protección integral de los derechos de niños, niñas y adolescentes en contexto de movilidad humana", mayo de 2020, 5, https://uni. $\mathrm{cf} / 3 \mathrm{~d} 1 \mathrm{ufmB}$.

3. Corte IDH, "Derechos y garantías de niñas y niños en el contexto de la migración y/o en necesidad de protección internacional", OC 21/14, 19 de agosto de 2014, párrs. 68 y 71.

4. Colombia, MRE, Decreto 216 de 2021, 1 de marzo de 2021. Colombia, UAEMC, Resolución 0971 de 2021, 28 de abril de 2021.

5. Filippo Grandi, "Discurso del Alto Comisionado de Naciones Unidas para los Refugiados sobre el nuevo Estatuto de Protección Temporal que Colombia otorgará a los venezolanos y venezolanas en el país", 8 de febrero de 2021, https://bit.ly/3j3SMeJ. 
Respecto a los niños migrantes venezolanos, el Estatuto contiene un capítulo específicamente dedicado a ellos. Allí se reconoce que contar con una situación migratoria regular es un paso previo y necesario para su protección integral y para prevenir la vulneración de sus derechos. En el presente trabajo, se parte del análisis de algunos de los mayores retos de protección de la niñez migrante en Colombia, para luego ahondar en el capítulo del Estatuto dedicado a niños y niñas venezolanos. Esto con el fin de preguntarse si este efectivamente responde a sus necesidades de protección y si podría ser un ejemplo para seguir en la región latinoamericana. En particular, se argumentará que el Estatuto representa un avance necesario pero insuficiente en este sentido, en cuanto contribuye a perpetuar una visión limitada y sesgada de la migración, la cual sigue permeando la política pública migratoria del Estado colombiano. Además, promueve la creación de un doble estándar de protección respecto a los niños de nacionalidades diferentes a la venezolana presentes en Colombia, que siguen sometidos al régimen migratorio ordinario. Finalmente, este texto es una invitación a seguir reflexionando sobre los mínimos innegociables de una política pública migratoria con enfoque de niñez, lo cual es una tarea pendiente en la región.

\section{RETOS DE PROTECCIÓN DE LA NIÑEZ EN SITUACIÓN DE MOVILIDAD HUMANA EN COLOMBIA}

Los datos sobre personas migrantes y, todavía más, sobre niños migrantes y refugiados son inadecuados e insuficientes. ${ }^{6}$ Esto, dada su vulnerabilidad y dependencia del Estado, tiene consecuencias de la mayor importancia en el diseño de la política pública y se refleja en la falta o limitación de la protección que reciben. ${ }^{7}$ Aun así, los datos disponibles nos permiten tener una imagen, aun desdibujada, de las dimensiones de la migración de la niñez. En 2015, el Fondo de Naciones Unidas para la Infancia (UNICEF) estimó que había 31 millones de niños y niñas migrantes sobre un total de 224 millones de personas migrantes. Esto significa decir que 1 entre 8 personas migrantes era un menor de edad. ${ }^{8}$ El Alto Comisionado de Naciones Unidas para los Refugiados (ACNUR), en 2020, estimó que el $41 \%$ de las personas desplazadas

\footnotetext{
6. ONU, UNICEF, Uprooted: The Growing Crisis for Refugee and Migrant Children, septiembre 2016, 15 y 115, https://bit.ly/2SSMBzs. Jacqueline Bhabha y Guy Abel, "Children and unsafe migration", en World Migration Report 2020, International Organization for Migration (IOM), 2019, 237.

7. Ann Singleton, "Data: creating the empirical base for development of child migration policy and protection", en Research Handbook on Child Migration, ed. Jacqueline Bhabha, Jyothi Kanics y Daniel Senovi1la Hernández (Cheltenham: Edward Elgar Publishing, 2018), 334.

8. ONU, UNICEF, Uprooted, 17.
} 
internacionalmente eran niños y que, entre 2018 y 2020, cerca de 1 millón de niños nacieron como personas refugiadas. ${ }^{9}$

Respecto a la población venezolana en particular, según datos de la plataforma de coordinación interagencial para refugiados y migrantes de Venezuela (R4V), cerca de 5,6 millones de nacionales venezolanos han salido de su país. De los 4,6 millones que se encuentran en países de América Latina y el Caribe, 1700000 se encuentran en Colombia, siendo este el país de destino de la mayoría de estos nacionales a nivel regional y global. ${ }^{10}$ Respecto a los niños, la autoridad migratoria del Estado colombiano - la Unidad Administrativa Especial Migración Colombia (UAEMC) - estimaba que, al 31 de diciembre de 2020, alrededor de 405000 niños venezolanos se encontraban en territorio colombiano, lo cual corresponde al $23 \%$ del total de nacionales venezolanos presentes en este país. ${ }^{11}$

Ya en 2018, cuando se llevó a cabo un primer intento de censar a la población venezolana en situación migratoria irregular presente en Colombia, se evidenció que el 27,4\% eran menores de edad. ${ }^{12}$ En esta ocasión, se ponía de relieve que el número de los niños venezolanos que habían sido atendidos en el marco de los programas del Instituto Colombiano de Bienestar Familiar (ICBF) había incrementado notablemente desde el año 2015. ${ }^{13}$ Así mismo, se subrayaba que el marco normativo era inadecuado y que las instituciones encargadas de la atención a la población migrante menor de edad carecían de información y conocimiento para responder de forma adecuada a sus necesidades, sobre todo, cuando se trataba de niños no acompañados y en situación migratoria irregular. Así las cosas, se hacía un llamado a la adecuación de las rutas de atención y estrategias para atender a esta población y a la creación de mecanismos para su acceso a una situación migratoria regular. ${ }^{14}$

9. ONU, ACNUR, Tendencias Globales. Desplazamiento Forzado en 2020, junio 2021, 10-1, https://bit. ly/3A4UCBW.

10. ONU, ACNUR y OIM, "Plataforma Regional de Coordinación Interagencial para Refugiados y Migrantes de Venezuela", https://www.r4v.info/es.

11. Colombia, UAEMC, Distribución Venezolanos en Colombia corte a 31 de diciembre, 29 de enero de 2021, https://bit.ly/3iXZi6F.

12. Se trata del Registro Administrativo para Migrantes Venezolanos (RAMV) que se realizó entre los meses de abril y junio de 2018. Este registro no consideró quienes ya contaban con un estatus migratorio regular en Colombia y tampoco reportó datos relativos a niños migrantes no acompañados. Colombia, Presidencia de la República, Decreto 542 de 2018, 21 de marzo de 2018.

13. El Instituto Colombiano de Bienestar Familiar es la entidad del Estado colombiano encargada de velar por los derechos de la población menor de edad presente en territorio colombiano, sin distinción de nacionalidad.

14. Colombia, Consejo Nacional de Política Económica y Social, Documento CONPES 3950. Estrategia para la atención de la migración desde Venezuela, 23 de noviembre de 2018, 54. 


\section{LA RESPUESTA DEL ESTADO COLOMBIANO ANTE LOS RETOS DE PROTECCIÓN DE LOS NIÑOS MIGRANTES VENEZOLANOS}

El Estado colombiano ha respondido a este llamado con diversas medidas, avanzando, entre otros, respecto a la garantía del derecho a la educación y encontrando dificultades en relación con la garantía del derecho a la salud y la regularización migratoria de los niños migrantes. Claro está que estos son solo algunos entre los aspectos relevantes para comprender la forma en que Colombia ha respondido a los desafíos antes señalados. Sin embargo, se pueden considerar ejemplificativos de los avances, al tiempo que de los obstáculos que todavía permanecen para la protección integral de la niñez migrante venezolana en territorio colombiano.

Respecto a la garantía del derecho a la educación, desde el año 2015, el Estado colombiano ha impulsado el ingreso de los niños de nacionalidad venezolana al sistema educativo. En particular, se ha establecido que deberá garantizarse la educación inicial, preescolar, básica y media a todos los niños procedentes de Venezuela presentes en el territorio. Para ello, no se podrá negar la posibilidad de matricularse a aquellos niños y niñas que no cuenten con un documento de identificación válido en Colombia y se encuentren en situación migratoria irregular. ${ }^{15}$ En este sentido, se estableció que la autoridad migratoria se abstendrá de iniciar un procedimiento administrativo sancionatorio en contra de las instituciones educativas que los matriculen, aunque no deja de requerirse que los menores queden registrados en el Sistema de Información para el Reporte de Extranjeros (SIRE) y que los padres se comprometan a regularizar su situación migratoria lo antes posible. ${ }^{16}$ Igualmente, se orienta respecto a la convalidación de estudios, a la validación de grados y a las estrategias de permanencia, tales como el transporte escolar y el acceso a los planes de alimentación escolar. ${ }^{17}$

A pesar de estos esfuerzos, los retos en materia de acceso y goce del derecho a la educación por parte de esta población permanecen. En 2020 se estimó que 250000 menores venezolanos no habían ingresado a ninguna institución educativa, lo que

15. Colombia, MEN y UAEMC, Circular Conjunta 016 de 2018, 10 de abril de 2018, https://bit.ly/3xY6B2k. Véase también, Colombia, Corte Constitucional, "Sentencia", T-185, 15 de junio de 2021.

16. Esto es problemático a la luz de la recomendación que entre instituciones educativas y autoridades migratorias no haya traspaso de información sobre la población migrante. ONU, Comité de los Derechos del Niño y Comité de los Derechos del Niño los Trabajadores Migratorios y de sus Familiares, Observación general conjunta núm. 3 (2017), párr. 17.

17. Silvia Ruiz Mancera, Lucía Ramírez Bolívar y Valentina Rozo Ángel, Acceso, promoción y permanencia de niños, niñas y adolescentes migrantes en el sistema educativo colombiano. Avances, retos y recomendaciones (Bogotá: Editorial Dejusticia, 2020), 42-5, https://bit.ly/2SqRI9O. 
en su momento equivalía a los $2 / 3$ de los niños venezolanos en Colombia. ${ }^{18}$ En este sentido, se advierte que la falta de un sustento mínimo que garantice la subsistencia del núcleo familiar, así como los costos indirectos de la educación, inhiben el acceso y la permanencia de los menores en las instituciones educativas. ${ }^{19}$ De igual manera, a pesar de haberse eliminado las barreras jurídicas para el acceso, la implementación de las normas es incoherente, lo cual resulta en la imposición de requisitos no previstos y en barreras ulteriores para el acceso y goce del derecho a la educación. Todo ello, aunado al hecho que los niños en situación migratoria irregular no pueden graduarse hasta no regularizar su situación migratoria, así como no pueden acceder a educación superior ni a formación profesional. ${ }^{20}$

Con relación a la garantía del derecho a la salud, las dificultades experimentadas por los niños migrantes para acceder a una atención integral muestran, de forma inequivocable, cómo la irregularidad migratoria afecta su acceso a derechos y servicios. ${ }^{21}$ En Colombia, a pesar de que la salud es un derecho fundamental para los niños, ${ }^{22}$ para la persona migrante el tipo de atención en salud al cual tendrá derecho depende de su estatus migratorio. Así, menores y adultos que se encuentren en situación migratoria regular deberán afiliarse al sistema de seguridad social y tendrán derecho a una atención integral en salud. De lo contrario, quienes estén en situación migratoria irregular recibirán únicamente atención básica y de urgencias ${ }^{23} \mathrm{y}$ accederán a la oferta de salud pública, hasta no regularizar su situación migratoria. De este régimen quedan exceptuados únicamente los recién nacidos, los cuales deberán ser afiliados al sistema de seguridad social independientemente de la situación migratoria

18. ONU, UNESCO, Derecho a la educación bajo presión. Principales desafíos y acciones transformadoras en la respuesta educativa al flujo migratorio mixto de población venezolana en Colombia, 2020, 27, https://bit.ly/3wS38SW.

19. Ibíd., 58. Child Resilience Alliance y UNICEF, Impacto del flujo migratorio de NNA venezolanos en el sistema educativo colombiano, 24 de abril de 2020, 9-10, https://bit.ly/3dsXIpV.

20. Juan Manuel Amaya-Castro, Carolina Moreno y Gracy Pelacani, La gestión de la migración en Colombia hoy: propuesta de diálogo para una política pública con enfoque de derechos en educación y salud, Serie: Informes CEM, Informe n. ${ }^{\circ}$ 01-2019, agosto de 2019, 25, https://bit.ly/3qwm1sl.

21. En este caso se entiende por derecho a la salud no solo la atención en salud sino también el "disfrute de toda una gama de facilidades, bienes, servicios y condiciones necesarios para alcanzar el más alto nivel posible de salud". ONU, Comité de Derechos Económicos, Sociales y Culturales, Observación General n. ${ }^{o} 14,11$ de agosto de 2000, E/C.12/2000/4, párr. 9, https://bit.ly/3zWPhwn.

22. Colombia, Constitución Política de la República de Colombia, Gaceta Constitucional n. ${ }^{\circ} 116,20$ de julio de 1991, art. 44.

23. En la sentencia T-210 de 2018, la Corte Constitucional de Colombia afirmó que la atención en urgencias debe tener un enfoque de derechos humanos y debe ser considerada de manera integral. Colombia, Corte Constitucional, "Sentencia", T-210, 1 de junio de 2018, s. p. 
de los padres, en aplicación del artículo 50 constitucional. ${ }^{24}$ En este sentido, no sobra recordar que se estima que más de la mitad de la población venezolana en Colombia se encuentra en situación migratoria irregular. ${ }^{25}$ Además, según datos de la Encuesta de Calidad de Vida de 2019 realizada por el Departamento Administrativo Nacional de Estadística, el $76 \%$ de los menores de edad migrantes resultó no estar afiliado al sistema de seguridad social. ${ }^{26}$

Sobre la protección del derecho a la salud de los niños migrantes venezolanos se ha pronunciado en varias ocasiones la Corte Constitucional colombiana, la cual, sobre todo desde 2017, cuenta con una jurisprudencia más extensa y variada en materia migratoria. ${ }^{27}$ Respecto a los menores migrantes, se trata de casos en los cuales los niños no habían sido afiliados al sistema de seguridad social por su situación migratoria irregular y/o no estaban recibiendo los tratamientos en salud que requerían, porque estos excedían la atención en urgencias. En sus pronunciamientos más recientes sobre este tema, la Corte reiteró que los menores de edad en situación migratoria irregular no deben ser responsabilizados o considerados culpables por los efectos adversos que derivan de esta situación, la cual no depende de ellos. ${ }^{28}$ Negar la atención en salud con base únicamente en la situación migratoria es desconocer el deber de actuar en garantía del interés superior de los niños. En conclusión, dice la Corte:

el Estado está en la obligación de prestar los servicios de salud a los menores de edad que sufren de algún tipo de afección física y mental y de garantizarles un tratamiento integral, adecuado y especializado conforme a la enfermedad padecida, incluyendo a los niños, niñas y adolescentes migrantes, a pesar de que no se encuentren regularizados en el país y, en consecuencia, no estén vinculados al SGSSS. ${ }^{29}$

Esta línea jurisprudencial muestra la relación intrínseca y problemática entre la regularidad migratoria y el acceso a derechos para la población migrante y para los niños, en particular. La dificultad o falta de acceso a mecanismos de regularización

24. Colombia, Constitución Política de la República, art. 50. Colombia, Ministerio de Salud y Protección Social, Decreto 780 de 2016, 6 de mayo de 2018, art. 2.1.3.10.

25. Colombia, UAEMC, Distribución de Venezolanos en Colombia - Corte 31 de enero de 2021, 3 de marzo de 2021, https://bit.ly/3qwvb7R.

26. Proyecto Migración Venezuela, "Caracterización de la niñez y adolescencia migrante en Colombia", Revista Semana, 23 de abril de 2021, 11, https://bit.ly/3h4PsgN.

27. Carolina Moreno V. y Gracy Pelacani, "Corte Constitucional colombiana: ¿un escenario posible para el Experimentalismo Constitucional en materia migratoria?", Latin American Law Review, n. ${ }^{\circ} 05$ (2020): 146-7, https://doi.org/10.29263/lar05.2020.07.

28. Colombia, Corte Constitucional, "Sentencia", T-178, 6 de mayo de 2019, s. p. Colombia, Corte Constitucional, "Sentencia", T-390, 7 de septiembre de 2020, s. p.

29. Colombia, Corte Constitucional, "Sentencia", T-090, 14 de abril de 2021, s. p. 
migratoria se debe a dos razones principales y es precisamente uno de los aspectos donde más se espera que impacte el recién adoptado Estatuto Temporal de Protección para Migrantes Venezolanos. La primera razón es la notoria dificultad, cuando no imposibilidad, para los nacionales venezolanos de acceder o renovar documentos oficiales, en particular, el pasaporte. ${ }^{30}$ La segunda razón está relacionada con los mecanismos de regularización migratoria predispuestos por el Estado colombiano para esta población.

Sobre este segundo aspecto y en línea con una cierta tendencia regional, ${ }^{31}$ Colombia creó en 2017 un permiso ad hoc para los nacionales venezolanos, los permisos especiales de permanencia (PEP). ${ }^{32}$ Para acceder a estos permisos era necesario, en primer lugar, haber ingresado de forma regular a territorio colombiano por un puesto de control migratorio autorizado, sellando el pasaporte. ${ }^{33}$ En segundo lugar, se necesitaba estar presente en el territorio a la fecha de publicación de la resolución que creaba o abría nuevos términos para aplicar a este permiso, así como se debía solicitarlo en los términos establecidos por la autoridad. ${ }^{34}$ No se trataba, por ende, de un mecanismo de regularización permanente, sino que dependía de la voluntad del Gobierno nacional abrir nuevos términos para el acceso, así como para la renovación del permiso.

El PEP, cuya expedición era gratuita, permitía acceder a la oferta institucional en materia de trabajo, educación, salud y atención de niños y adolescentes. Una vez obtenido el permiso, este era válido por un máximo de dos años, debiéndose renovar

30. CIDH, Resolución 2/18, Migración Forzada de Personas Venezolanas, 2 de marzo de 2018, https://bit. 1y/3gSBFux; ONU, Alto Comisionado de las Naciones Unidas para los Derechos Humanos, Informe de la Alta Comisionada de las Naciones Unidas para los Derechos Humanos sobre la situación de los derechos humanos en la República Bolivariana de Venezuela, 12 de julio de 2019, A/HRC/41/18.

31. Diego Acosta, Cécile Blouin y Luisa Feline Freier, La emigración venezolana: respuestas latinoamericanas, Documentos de trabajo 3/2019, Fundación Carolina, 2019, 8-11, https://bit.ly/35TLaDw. Andrew Selee, Jessica Bolter, Betilde Muñoz-Pogossian y Miryam Hazán, Creatividad dentro de la crisis: opciones legales para migrantes venezolanos en América Latina, Migration Policy Institute, Organización de Estados Americanos, 31 de enero de 2019, 7-11, https://bit.ly/3Ack4Fw.

32. El PEP se creó en 2017 y en seis ocasiones se abrieron nuevos términos para acceder a este permiso y para su renovación. Colombia, MRE, Resolución 5797 de 2017, 25 de julio de 2017. Así mismo, en 2020 se creó un permiso especial de permanencia para el fomento de la formalización (PEPFF) que no requería pasaporte ni haber ingresado regularmente, pero sí ser destinatario de una oferta de trabajo. Colombia, Ministerio del Trabajo, Decreto 117 de 2020, 28 de enero de 2020.

33. El pasaporte podía estar vencido hasta por dos años. El 9 de junio de 2021 se expidió una nueva resolución que permite el uso de pasaportes venezolanos vencidos hasta por un término de diez años siguientes a la fecha de vencimiento o de prórroga para ingresar, transitar, permanecer y salir del territorio colombiano, así como para solicitar los permisos pertinentes. Colombia, MRE, Resolución 2231 de 2021, 9 de junio de 2021.

34. Se requiere también no tener antecedentes judiciales a nivel nacional e internacional, así como no tener una medida de deportación o expulsión vigente. Colombia, MRE, Resolución 5797 de 2017, art. 1. 
transcurrido este término. Se trataba de un permiso individual que no permitía extender a otros beneficiarios, como los miembros del núcleo familiar del titular, el acceso a derechos y servicios asociados al PEP o regularizar su situación migratoria. Así mismo, no permitía acumular tiempo de residencia en el país con el fin de acceder a un estatus más estable y permanente.

Así las cosas, no contar con un pasaporte, haber ingresado al territorio fuera de los términos establecidos o no haber solicitado a tiempo el permiso, impedía acceder a él. Para los niños no se preveía ninguna excepción o trato preferencial, imponiéndoles cumplir con los mismos requisitos previstos para la población adulta. A partir del mes de octubre de 2019, el estimado de los nacionales venezolanos en situación migratoria irregular fue mayor al de los que se encontraba en situación migratoria regular. Es en este contexto y para responder a estos desafíos que, en marzo de 2021, se adopta el ETPV.

\section{EL ENFOQUE DE NIÑEZ DEL ESTATUTO TEMPORAL DE PROTECCIÓN PARA MIGRANTES VENEZOLANOS}

El Estatuto contempla de forma explícita las necesidades específicas de niños venezolanos en Colombia, exceptuándolos de tener que cumplir con ciertos requisitos previstos para la población adulta y previendo un trato más favorable para ellos en relación con el acceso a un estatus migratorio regular. En especial, el Decreto por medio del cual se adopta el Estatuto y la resolución que lo implementa ${ }^{35}$ afirman que, con base en las obligaciones internacionales asumidas por Colombia como Estado parte de la Convención de los Derechos del Niño, se deberán adoptar todas las medidas administrativas, legislativas o de otro tipo para dar efectividad a los derechos reconocidos en la Convención. ${ }^{36}$ Además, se establece que las disposiciones de la resolución se interpretarán de la forma más favorable para el interés superior y protección integral de niños migrantes. Así mismo, se recuerda que el Código de Infancia y Adolescencia, el principal cuerpo normativo que a nivel nacional establece las medidas de protección de los menores de edad en Colombia, se aplica a todos los niños y adolescentes presentes en el territorio colombiano, independientemente de su nacionalidad y estatus migratorio, de conformidad con el principio de no discriminación. ${ }^{37}$

35. El título IV de la Resolución 0971 de 2021 que implementa el ETPV está dedicado específicamente a la aplicación del Estatuto a la situación de niños, niñas y adolescentes venezolanos.

36. Colombia, Ley 12 de 1991, Diario Oficial n. ${ }^{\circ}$ 39.640, 22 de enero de 1991.

37. Colombia, Ley 1098 de 2006, Diario Oficial n. ${ }^{\circ} 46.446,8$ de noviembre de 2006, art. 4. 
En particular, el Estatuto reconoce que la situación migratoria regular es esencial para asegurar una protección integral, la prevención de vulneraciones y la garantía de los derechos a los niños migrantes. En este contexto, se deja claro que los niños no acompañados y separados son especialmente vulnerables. De igual manera, se menciona que para los menores que se encuentran en un proceso administrativo de restablecimiento de derechos (PARD) o en el sistema de responsabilidad penal adolescente (SRPA), es decir, bajo la protección del Estado, es fundamental contar con una situación migratoria regular, con documentos de identificación y con la garantía de poder acceder a todos los servicios del Estado.

Sin embargo, para comprender la forma en que este enfoque diferencial se materializa, es necesario delinear brevemente qué prevé el ETPV. Este se compone de dos elementos: el Registro Único de Migrantes Venezolanos (RUMV o registro), el cual comprende datos de carácter biográfico, demográfico y biométrico, y el Permiso por Protección Temporal (PPT), que se otorgará únicamente a quienes hayan realizado previamente el registro. Para el acceso a ambos se establecen ventanas de tiempo predeterminadas. La persona que no realice el registro o no solicite el permiso dentro de estos términos estará sujeta a los procedimientos administrativos sancionatorios de carácter migratorio a los que haya lugar.

Respecto a los niños, se establece que estos deberán cumplir con alguna de las siguientes condiciones para acceder al registro y al permiso, las cuales son idénticas a las que tiene que cumplir la población adulta. Las condiciones son: encontrarse en situación migratoria regular como titular de un PEP, de un permiso de breve estadía o del documento que comprueba que es solicitante de reconocimiento de la condición de refugiado; demostrar que se encontraba en territorio colombiano en situación migratoria irregular al 31 de enero de 2021; o ingresar de forma regular al territorio en los primeros dos años de vigencia del Estatuto. Así mismo, solo tendrán acceso al registro quienes, sean mayores o menores de edad, se encuentren en territorio colombiano, puedan presentar un documento de identificación vigente o vencido, ${ }^{38}$ declaren su intención de permanecer temporalmente en Colombia, autoricen la recolección de sus datos ${ }^{39} \mathrm{y}$ aporten prueba sumaria de su presencia en el territorio de forma irregular al 31 de enero de 2021, si este es el caso.

38. Estos son el pasaporte, el acta de nacimiento venezolana, la cédula de ciudadanía venezolana y el permiso especial de permanencia. Colombia, UAEMC, Resolución 0971 de 2021, art. 5.

39. Los datos biométricos se recolectarán únicamente respecto a los menores de edad de 7 o más años de edad. La Resolución 0971 establece que los niños bajo protección del Estado y aquellos que no hayan cumplido sus estudios de educación media, pero no hayan podido acceder a su título de bachillero por encontrarse en situación migratoria irregular, serán priorizados para la recolección de sus datos biométricos y para la expedición del PPT. Ibíd., art. 33. 
Ahora bien, aunque se prevén términos específicos para realizar el registro, para los niños vinculados a un PARD o al SRPA, y para quienes estén matriculados en una institución educativa en los niveles inicial, prescolar, básica y media, el registro podrá realizarse durante toda la vigencia del Decreto que adopta el Estatuto, es decir hasta el mes de mayo de 2031.

El registro de niños y adolescentes lo realizará el padre o la madre, o los otros miembros de su familia o adultos responsables que lo acompañan. Estos deberán aportar el documento de identidad del menor y un documento que da cuenta del parentesco o que otorga el cuidado o custodia del menor. Cuando no se puedan aportar estos documentos, deberá informarse de ello a la autoridad migratoria. Esta, si evidencia una presunta amenaza o vulneración de los derechos del niño, informará a la autoridad administrativa competente - el ICBF — que hará las verificaciones oportunas. De no comprobarse alguna amenaza o vulneración a los derechos del menor, se seguirá con el trámite de registro y otorgamiento del permiso, sin que la falta de los documentos mencionados sea obstáculo para ello, permitiendo de esta forma el acceso al Estatuto a aquellos niños que estén indocumentados. Cuando los menores se encuentren bajo protección del Estado, la autoridad administrativa adelantará el registro, debiendo aportar únicamente copia del documento que da cuenta del ingreso del niño al proceso administrativo, de la medida de internamiento o sanción judicial.

Una vez cumplidas estas condiciones y requisitos, se solicitará el otorgamiento de un PPT a beneficio del niño, ${ }^{40}$ siempre y cuando esté incluido en el registro, no haya sido reconocido como refugiado o tenga una solicitud vigente de protección internacional vigente en otro país. ${ }^{41}$ De ser autorizado el permiso, su entrega se hará en las mismas modalidades y tiempos establecidos para la población adulta. Este permiso tendrá una vigencia de diez años y permitirá a su titular estar en situación migratoria regular, acceder al sistema de seguridad social en salud y pensión, acceder a los niveles de educación inicial, preescolar, básica, media y superior y a la formación profesional y ejercer cualquier actividad de ocupación legal, de ser el caso. No sobra añadir que este permiso permite acumular tiempo de residencia en el país para, eventualmente, acceder a una visa como residente, de cumplirse los demás requisitos.

40. Esto es así porque el otorgamiento del permiso es una facultad potestativa y discrecional de la autoridad migratoria. Por ende, el cumplimiento de todos los requisitos previstos no es garantía para su otorgamiento. Colombia, MRE, Decreto 216 de 2021, art. 12, párr. 2.

41. A los mayores de edad se les requiere, además, no tener antecedentes penales, anotaciones o procesos administrativos o judiciales en curso en Colombia o en el exterior; no tener en curso investigaciones administrativas migratorias; no tener en su contra medidas de expulsión, deportación o sanción económica vigentes. Colombia, UAEMC, Resolución 0971 de 2021, art. 15. 
Finalmente, respecto a las causales de cancelación del permiso, en caso de los niños esto ocurrirá únicamente cuando el menor se ausente del territorio nacional por un período superior a los ciento ochenta días calendario continuos. Ni siquiera si comete una infracción al ordenamiento jurídico y por ello ingresa al SRPA se cancelará por ello su permiso. Este aspecto es de extrema relevancia, ya que se determina que un niño o adolescente, por su condición, no puede incurrir en infracción migratoria, su presencia no se podrá considerar inconveniente para el país o un riesgo para la seguridad nacional o ciudadana, así como no podrá ser responsabilizado por falsedades o inconsistencias en los documentos presentados. Todas ellas sí serán causales de cancelación del PPT para los mayores de edad.

\section{NECESARIO, PERO NO SUFICIENTE. CRÍTICAS AL ESTATUTO TEMPORAL DE PROTECCIÓN}

El ETPV representa, sin duda, un avance necesario en la política pública migratoria del Estado colombiano respecto a la población venezolana. Sobre todo con relación a la regularización migratoria, el enfoque de niñez que presenta el Estatuto es fundamental para la garantía de los derechos de esta población. Sin embargo, cabe preguntarse si este realmente responde a las necesidades de los niños venezolanos en Colombia. En este sentido, hay por lo menos tres aspectos con base en los cuales se afirma que el ETPV es insuficiente y perpetua una visión sesgada de la migración venezolana en territorio colombiano.

En primer lugar, hay que recordar que el Estatuto presenta un ámbito de aplicación temporal y personal limitados. A pesar de que tenga una vigencia de diez años, se establece que el Gobierno nacional podrá prorrogarlo pero, sobre todo, dar por terminados sus efectos en cualquier momento, con base en sus facultades discrecionales. ${ }^{42}$ Aún más relevante es que excluye de su ámbito de aplicación personal a aquellos niños que hayan ingresado a territorio colombiano de forma irregular después del 31 de enero de 2021, con el objetivo de incentivar la migración regular. Así mismo, excluye a los menores que no puedan demostrar que se encontraban en territorio colombiano de forma irregular a la misma fecha. De esta forma desconoce las razones que obligan esta población al ingreso irregular y que explican su falta de documentación, ${ }^{43}$ así

42. Gracy Pelacani et al., Comentarios al proyecto de decreto que crea el Estatuto Temporal de Protección para Migrantes Venezolanos en Colombia: Hoy más vigentes que nunca ad portas de la reglamentación (Bogotá D. C.: Universidad de los Andes, 2021), https://bit.ly/3gZfdjr.

43. Véase, entre otros, CIDH, Informe Anual 2019, Capitulo IV: Venezuela, 24 de febrero de 2020, OEA/ Ser.L/V/II, Doc. 5, párrs. 434 y siguientes. 
como perpetúa una visión sesgada e ingenua de la migración irregular, dando paso a la ulterior vulneración de sus derechos. Esta visión ingenua predica que la migración irregular es fruto de una decisión voluntaria y consciente de la persona migrante, la cual deberá, por ende, asumir la responsabilidad del ingreso irregular, como la de ser excluida del Estatuto. ${ }^{44}$ En este sentido, respecto a los niños contraría la posición de la Corte Constitucional, la cual ha sido clara en afirmar que los menores de edad migrantes no deben ser responsabilizados o considerados culpables de su situación de irregularidad migratoria, la cual no depende de ellos. Este aspecto es aún más importante, si se piensa que el Estado colombiano ha dejado de expedir permisos especiales de permanencia desde la adopción del Estatuto. Es decir, el Estatuto es el principal canal para el acceso a una situación migratoria regular para esta población y el único cuando no se cuente con un pasaporte. ${ }^{45}$

En segundo lugar, el PPT sigue siendo un permiso individual, cuyos beneficios no son extensibles al núcleo familiar del titular. Así, en el caso en que el niño cumpla con los requisitos para acceder el Estatuto, pero otros miembros de su núcleo familiar no los cumplan, quedarán excluidos. De la misma forma, los niños no acompañados o separados que accedan al Estatuto no podrán extender sus beneficios a los padres, si ellos no cumplen con los requisitos establecidos para la población adulta, en caso de ser posible ubicar a la familia de origen y la sucesiva reunificación familiar en Colombia. Este aspecto sorprende aún más en el caso del Estatuto, ya que el PPT permite acumular tiempo de residencia en el país para acceder a una visa como residente y que el otro permiso cuya titularidad tiene el mismo efecto — la visa como migrante — sí permite al titular tener beneficiarios en su visa. Así mismo, se permite a los solicitantes de reconocimiento de la condición de refugiado asociar a su solicitud miembros de su núcleo familiar, los cuales accederán a un salvoconducto que regularizará su situación y les permitirá afiliarse al sistema de seguridad social en salud.

Así, con la insistencia en un permiso individual, se perpetúa nuevamente una visión sesgada de la migración, la cual estaría constituida por individuos singularmente considerados que viajan solos y pretenden quedarse solos en el país de destino. Respecto a los niños migrantes, en particular, se insiste en el desarrollo de medidas que ignoran que la protección del núcleo familiar del menor es parte fundamental de las

44. Elisa Ortega Velásquez, "Los niños migrantes irregulares y sus derechos humanos en la práctica europea y americana: entre el control y la protección", Boletín Mexicano de Derecho Comparado, nueva serie, año XLVIII, n. ${ }^{\circ} 142$ (enero-abril 2015): 198-200.

45. Aunque el documento que se otorga a quienes soliciten el reconocimiento de la condición de refugiado regulariza la situación migratoria del solicitante, este no es, en estricto sentido, un mecanismo de regularización migratoria. 
medidas de protección de la niñez migrante. ${ }^{46}$ Así mismo, se desconocen los efectos adversos para el acceso y garantía de los derechos de los niños que puede tener ser parte de un núcleo familiar en situación migratoria irregular. ${ }^{47}$

En tercer y último lugar, es importante subrayar que el Estatuto es una medida accesible únicamente a los nacionales venezolanos. Los niños extranjeros de otras nacionalidades que se encuentren en similares situaciones de vulnerabilidad y en la imposibilidad, entre otras, de acceder a un estatus migratorio regular por razones ajenas a su voluntad, deberán someterse al régimen migratorio ordinario, que no prevé ninguna medida específica en beneficio de los menores de edad migrantes. Así, se promueve y justifica la creación de un doble estándar de tratamiento, el cual excluye e invisibiliza a los niños extranjeros de otras nacionalidades que quedan a los márgenes de la política y agenda pública en materia migratoria. Así mismo, se perpetua una visión limitada de la migración a territorio colombiano, que no está compuesta únicamente por la migración de personas venezolanas ${ }^{48}$ y se desconoce que:

al diseñar, adoptar e implementar sus políticas migratorias relativas a personas menores de 18 años de edad, los Estados deben priorizar el enfoque de los derechos humanos desde una perspectiva que tenga en cuenta en forma transversal los derechos de niñas y niños y, en particular, su protección y desarrollo integral, los cuales deben primar por sobre cualquier consideración de la nacionalidad o el estatus migratorio, a fin de asegurar la plena vigencia de sus derechos. ${ }^{49}$ (énfasis añadido)

\section{CONCLUSIONES}

Niños y niñas son particularmente vulnerables en contextos de migración internacional, aún más cuando ellos y/o sus familias se encuentran en situación migratoria irregular. Por ello, las políticas migratorias de los Estados necesitan incluir un enfoque

46. Corte IDH, Caso Familia Pacheco Tineo vs. Estado Plurinacional de Bolivia (Excepciones Preliminares, Fondo, Reparaciones y Costas), 25 de noviembre de 2013, Serie C n. ${ }^{\circ} 272$, párrs. 217-20.

47. CIDH, ¿Cómo hacer más efectiva la protección a la unidad familiar y la reunificación familiar en situaciones de movilidad humana y movimientos mixtos, y en contexto de pandemia?, 2021, https://bit. ly/35WFVD9.

48. UNICEF informó que en los últimos cuatro años la migración de niños a través de la provincia del Darién hacia Panamá se ha multiplicado por 15 , siendo los niños el $25 \%$ de la población que utiliza esta ruta migratoria notoriamente peligrosa. ONU, UNICEF, Quince veces más niños, niñas y adolescentes cruzan la selva de Panamá hacia Estados Unidos en los últimos cuatro años, 29 de marzo de 2021, https://uni. $\mathrm{cf} / 3 \mathrm{y} 0 \mathrm{oKN} 3$.

49. Corte IDH, "Derechos y garantías de niñas y niños en el contexto de la migración y/o en necesidad de protección internacional”, OC 21/14, 19 de agosto de 2014, párr. 68. 
de niñez que considere que los niños migrantes son, ante todo, niños, y que no deberán ser responsabilizados ni culpabilizados por su situación migratoria ni por la de sus padres. En este contexto, el Estatuto Temporal de Protección para Migrantes Venezolanos, adoptado en marzo de 2021, pretendió responder a algunos de los vacíos de la política migratoria colombiana respecto a la población venezolana, primordialmente en relación con el acceso a un estatus migratorio regular y a la falta de datos sobre esta población. En particular, el Estatuto incluyó medidas específicas en beneficio de niños venezolanos, con el objetivo de facilitar su regularización migratoria y el acceso a derechos y servicios.

Antes de la adopción del Estatuto, el Estado colombiano había adoptado una serie de medidas para responder a los retos en materia de protección de los niños venezolanos que arriban a territorio colombiano. Entre otros, ha adoptado medidas para promover la protección de su derecho a la educación, garantizando su ingreso a las instituciones educativas a pesar de su estatus migratorio irregular. Sin embargo, permanecen varios obstáculos para la garantía del derecho a recibir atención integral en salud cuando se encuentran en situación migratoria irregular, así como para acceder a mecanismos de regularización migratoria.

El Estatuto recién adoptado pretende responder a estos desafios a través de la previsión de medidas específicas para el acceso de los niños venezolanos a un estatus migratorio regular. Esto, en el entendido de que la regularidad migratoria es el paso previo e ineludible para la garantía de sus derechos y protección integral. Sin embargo, el Estatuto, aun representando un avance importante en la política pública migratoria del Estado colombiano, es insuficiente por al menos tres motivos. El primero es que excluye de su ámbito de aplicación a aquellos niños y niñas que ingresen de forma irregular a territorio colombiano después del 31 de enero de 2021 o que no puedan demonstrar que se encontraban en el territorio de forma irregular antes de esta fecha. El segundo es que no contempla la protección de la familia del menor de edad migrante como unos de sus objetivos, ya que no posibilita la extensión del permiso obtenido y de los derechos a los que este da acceso a los miembros del núcleo familiar del menor. El tercer y último motivo es que promueve y justifica la creación de un doble estándar de protección en la política pública migratoria colombiana, la cual excluye a los niños de otras nacionalidades que pueden encontrarse en una condición de vulnerabilidad similar. Así, aunque el Estatuto da pautas importantes que seguir en el desarrollo de una política pública migratoria con enfoque de niñez, es solo un primer paso de un largo camino que todavía queda por recorrer. 


\section{BIBLIOGRAFÍA}

Acosta, Diego, Cécile Blouin y Luisa Feline Freier. La emigración venezolana: respuestas latinoamericanas. Documentos de trabajo 3/2019. Fundación Carolina, 2019. https://bit. ly/35TLaDw.

Amaya-Castro, Juan Manuel, Carolina Moreno y Gracy Pelacani. La gestión de la migración en Colombia hoy: Propuesta de diálogo para una política pública con enfoque de derechos en educación y salud. Serie: Informes CEM. Informe n. ${ }^{\circ}$ 01-2019. Agosto 2019. https://bit. ly/3qwm1sl.

Bhabha, Jacqueline, y Guy Abel. "Children and unsafe migration". En World Migration Report 2020. International Organization for Migration (IOM), 2019.

Child Resilience Alliance y UNICEF. Impacto del flujo migratorio de NNA venezolanos en el sistema educativo colombiano. 24 de abril de 2020. https://bit.ly/3dsXIpV.

CIDH. ¿Cómo hacer más efectiva la protección a la unidad familiar y la reunificación familiar en situaciones de movilidad humana y movimientos mixtos, y en contexto de pandemia? 2021. https://bit.ly/35WFVD9.

—.Informe Anual 2019. Capítulo IV: Venezuela. 24 de febrero de 2020. OEA/Ser.L/V/II, Doc. 5 .

- Resolución 2/18. Migración Forzada de Personas Venezolanas. 2 de marzo de 2018. https://bit.ly/3gSBFux.

Colombia. Consejo Nacional de Política Económica y Social. Documento CONPES 3950. Estrategia para la atención de la migración desde Venezuela. 23 de noviembre de 2018.

Colombia. Constitución Política de la República de Colombia. Gaceta Constitucional n. ${ }^{\circ} 116$, 20 de julio de 1991.

Colombia. Corte Constitucional. "Sentencia". T-210, 1 de junio de 2018.

—. "Sentencia". T-178, 6 de mayo de 2019.

—. "Sentencia". T-390, 7 de septiembre de 2020.

—. "Sentencia". T-090, 14 de abril de 2021.

—. "Sentencia". T-185, 15 de junio de 2021.

Colombia. Ley 1098 de 2006. Diario Oficial n. ${ }^{\circ} 46.446,8$ de noviembre de 2006.

-. Ley 12 de 1991. Diario Oficial n. ${ }^{\circ}$ 39.640, 22 de enero de 1991.

Colombia. MEN y UAEMC. Circular Conjunta 016 de 2018. 10 de abril de 2018. https://bit. ly/3xY6B2k.

Colombia. Ministerio de Salud y Protección Social. Decreto 780 de 2016. 6 de mayo de 2018.

Colombia. Ministerio del Trabajo. Decreto 117 de 2020. 28 de enero de 2020.

Colombia. MRE. Resolución 2231 de 2021. 9 de junio de 2021.

—. MRE. Resolución 5797 de 2017. 25 de julio de 2017.

—. MRE. Decreto 216 de 2021. 1 de marzo de 2021. 
Colombia. Presidencia de la República. Decreto 542 de 2018. 21 de marzo de 2018.

Colombia. UAEMC. Distribución de Venezolanos en Colombia. Corte 31 de enero de 2021. 3 de marzo de 2021, https://bit.ly/3qwvb7R.

-. UAEMC. Distribución Venezolanos en Colombia. Corte a 31 de diciembre. 29 de enero de 2021. https://bit.ly/3iXZi6F.

—. UAEMC. Resolución 0971 de 2021. 28 de abril de 2021.

Corte IDH, "Derechos y garantías de niñas y niños en el contexto de la migración y/o en necesidad de protección internacional”, OC 21/14, 19 de agosto de 2014, Serie A, n. ${ }^{\circ} 21$.

-. Caso Familia Pacheco Tineo vs. Estado Plurinacional de Bolivia (Excepciones Preliminares, Fondo, Reparaciones y Costas). 25 de noviembre de 2013, Serie C, n. ${ }^{\circ} 272$.

Grandi, Filippo. "Discurso del Alto Comisionado de Naciones Unidas para los Refugiados sobre el nuevo Estatuto de Protección Temporal que Colombia otorgará a los venezolanos y venezolanas en el país". 8 de febrero de 2021. https://bit.ly/3j3SMeJ.

Moreno V., Carolina, y Gracy Pelacani. "Corte Constitucional colombiana: ¿un escenario posible para el Experimentalismo Constitucional en materia migratoria?”. Latin American Law Review, n. ${ }^{\circ} 5$ (2020): 139-57. https://doi.org/10.29263/lar05.2020.07.

ONU. ACNUR. Tendencias Globales. Desplazamiento Forzado en 2020. junio de 2021. https://bit.ly/3A4UCBW.

ONU. ACNUR y OIM. Plataforma Regional de Coordinación Interagencial para Refugiados y Migrantes de Venezuela. https://www.r4v.info/es.

ONU. Alto Comisionado de las Naciones Unidas para los Derechos Humanos. Informe de la Alta Comisionada de las Naciones Unidas para los Derechos Humanos sobre la situación de los derechos humanos en la República Bolivariana de Venezuela. 12 de julio de 2019. $\mathrm{A} / \mathrm{HRC} / 41 / 18$.

ONU. Comité de Derechos Económicos, Sociales y Culturales. Observación General n ${ }^{\circ} 14$. 11 de agosto de 2000. E/C.12/2000/4. https://bit.ly/3zWPhwn.

ONU. Comité de los Derechos del Niño y Comité de los Derechos del Niño los Trabajadores Migratorios y de sus Familiares. Observación general conjunta $n .^{\circ} 3$ (2017) del Comité de Protección de los Derechos de Todos los Trabajadores Migratorios y de sus Familiares y $n .^{\circ} 22$ (2017) del Comité de los Derechos del Niño sobre los principios generales relativos a los derechos humanos de los niños en el contexto de la migración internacional. 16 de noviembre de 2017. CMW/C/GC/3-CRC/C/GC/22.

ONU. UNESCO. Derecho a la educación bajo presión. Principales desafios y acciones transformadoras en la respuesta educativa al flujo migratorio mixto de población venezolana en Colombia. 2020. https://bit.ly/3wS38SW.

ONU. UNICEF. La regularización migratoria como condición esencial para la protección integral de los derechos de niños, niñas y adolescentes en contexto de movilidad humana. Mayo de 2020. https://uni.cf/3d1ufmB.

- Quince veces más niños, niñas y adolescentes cruzan la selva de Panamá hacia Estados Unidos en los últimos cuatro años. 29 de marzo de 2021. https://uni.cf/3y0oKN3. 
-. Uprooted: The Growing Crisis for Refugee and Migrant Children. Septiembre de 2016. https://bit.ly/2SSMBzs.

Ortega Velásquez, Elisa. "Los niños migrantes irregulares y sus derechos humanos en la práctica europea y americana: entre el control y la protección". Boletín Mexicano de Derecho Comparado, nueva serie, año XLVIII, n. ${ }^{\circ} 142$ (enero-abril 2015): 185-221.

Pelacani, Gracy, Carolina Moreno V., Laura Dib-Ayesta, Mairene Tobón, Laura Rojas, Estefanía Laborde, María Lucía Hernández y Allison Brooke Wolf. Comentarios al proyecto de decreto que crea el Estatuto Temporal de Protección para Migrantes Venezolanos en Colombia: Hoy más vigentes que nunca ad portas de la reglamentación. Bogotá D.C.: Universidad de los Andes, 2021. https://bit.ly/3gZfdjr.

Proyecto Migración Venezuela. “Caracterización de la niñez y adolescencia migrante en Colombia”. Revista Semana. 23 de abril de 2021. https://bit.ly/3h4PsgN.

Ruiz Mancera, Silvia, Lucía Ramírez Bolívar y Valentina Rozo Ángel. Acceso, promoción y permanencia de niños, niñas y adolescentes migrantes en el sistema educativo colombiano. Avances, retos y recomendaciones. Bogotá: Editorial Dejusticia, 2020. https://bit. ly/2SqRI9O.

Selee, Andrew, Jessica Bolter, Betilde Muñoz-Pogossian y Miryam Hazán. Creatividad dentro de la crisis: opciones legales para migrantes venezolanos en América Latina. Migration Policy Institute, Organización de Estados Americanos. 31 enero de 2019. https://bit. ly/3Ack4Fw.

Singleton, Ann. "Data: creating the empirical base for development of child migration policy and protection". En Research Handbook on Child Migration, editado por Jacqueline Bhabha, Jyothi Kanics y Daniel Senovilla Hernández. Cheltenham: Edward Elgar Publishing, 2018. 